\title{
Sense and Self-Regulation Checklist, a Measure of Comorbid Autism Symptoms: Initial Psychometric Evidence
}

\author{
Louisa M. T. Silva, Mark Schalock
}

KEY WORDS

- autistic disorder

- sensation

- sensation disorders

- sensory threshold

- signs and symptoms
Sensory and self-regulatory symptoms make up most of the comorbid symptoms in autism and are associated with increased autism severity. We validated a parent-caregiver measure of comorbid symptoms in autism, the Sense and Self-Regulation Checklist (SSC), in 265 children <6 yr with typical development ( $n=138)$, autism $(n=99)$, or other developmental delay $(n=28)$; Cronbach's $\alpha$ was .87. We report two new findings discriminating autism from other groups: (1) multifocal tactile sensory impairment, characterized by hyporeactivity to injurious stimuli and hyperreactivity to noninjurious stimuli $(F[2,262]=86.8$, $p<.001)$ and $(2)$ global self-regulatory delay $(F[2,262]=122, p<.001)$. Both findings suggest an explanation for social delay in children with autism. The SSC reports a prevalence of sensory and selfregulatory findings approaching $100 \%$ in the autism group (96\% and $98 \%$, respectively), raising the possibility that sensory and self-regulatory difficulties represent a core part of autism.

Silva, L. M. T., \& Schalock, M. (2012). Sense and Self-Regulation Checklist, a measure of comorbid autism symptoms: Initial psychometric evidence. American Journal of Occupational Therapy, 66, 177-186. http://dx.doi.org/10.5014/ ajot.2012.001578

Louisa M. T. Silva, MD, MPH, is Visiting Professor, Teaching Research Institute, Western Oregon University, P0 Box 688, Salem, OR 97308; Imtsilvaqigong@comcast. net

Mark Schalock is Associate Research Professor, Teaching Research Institute, Western Oregon University, Monmouth.

C omorbid symptoms are common in autism. Their importance to the function and outcome of the development of young children with autism is reflected in current recommendations to evaluate and treat comorbid symptoms at the time of autism diagnosis (Levy, Mandell, \& Schultz, 2009). The most common comorbid symptoms in young children with autism are abnormal sensory responses (90\%; Leekam, Nieto, Libby, Wing, \& Gould, 2007), sleep disruption (86\%; Liu, Hubbard, Fabes, \& Adam, 2006), and gastrointestinal disorders (70\%; Ibrahim, Voigt, Katusic, Weaver, \& Barbaresi, 2009; Valicenti-McDermott et al., 2006), followed by self-injurious behavior (34\%; Hartley, Sikora, \& McCoy, 2008) and aggression and irritability (22\%; Hartley et al., 2008). Why comorbid symptoms are so common, and how they might be related to core symptoms, is not well understood. However, comorbid symptoms are known to increase the severity of autism (Malow et al., 2006; Nikolov et al., 2009; Souders et al., 2009).

The most common comorbid symptom in autism is abnormal sensory responses. A considerable body of work has shown a mixed form of sensory threshold abnormality in young children with autism (Baranek, David, Poe, Stone, \& Watson, 2006; Ben-Sasson et al., 2009; Dunn, 1999), with both hyporeactivity and hyperreactivity to sensory stimuli present (Tordjman et al., 2009). This hyporeactivity and hyperreactivity are distinguished from those of typically developing or developmentally delayed children in that they are more common, more severe, and multisensory (Kern et al., 2006).

The remaining comorbid symptoms seen in autism are consistent with early symptoms of delayed self-regulation. Examining them relative to the developmental trajectory of self-regulation can be illuminating. In a typically 
developing child, self-regulation begins in infancy with physiological regulation of sleep-wake cycles, feeding, and self-soothing. The self-regulation of orientation and attention occurs between ages 3 and 7 mo and is the starting point for social learning. By age 3, the typically developing child can demonstrate emotional and behavioral self-regulation in response to social demands (Posner $\&$ Rothbart, 2009). The most common symptoms of failure to achieve self-regulation milestones in children presenting for clinical management are sleep and feeding difficulties; difficulty self-soothing; failure to orient to and attend to caregivers; and behavioral responses such as prolonged tantrums, aggression, and self-injurious behavior (Reebye \& Elbe, 2009). These symptoms constitute the majority of comorbid symptoms in autism. Given that the self-regulation milestones are a necessary foundation for early social, emotional, and cognitive development, it is of interest to examine how their delay might affect the core symptoms of autism.

For the past $10 \mathrm{yr}$, we have been involved in autism research evaluating a model and treatment of autism based on concepts of Chinese medicine that addresses both core and comorbid symptoms (Silva, Schalock, \& Ayres, 2011). The model proposes that the core symptoms of autism are secondary to a condition of disordered sensory and self-regulation. The Five-Phase Theory of Chinese medicine asserts that sensory and self-regulatory functions are profoundly interlinked; each individual sense is linked with a particular set of physiological functions (e.g., touch is linked with self-soothing, and taste is linked with digestion; Yanchi, 1988). As such, normal sensory function is required for the timely unfolding of selfregulation, and abnormal sensory function will result in self-regulatory delays. The treatment we have developed-a parent-delivered, staff-supported massage methodology based on Chinese medicine- has shown robust improvement of abnormal sensory responses and self-regulation, as well as improvement in measures of autism, in two randomized controlled trials (Silva, Schalock, Ayres, Bunse, \& Budden, 2009; Silva, Schalock, \& Gabrielsen, 2011). The research required the development of an outcomes measure for sensory and self-regulatory symptoms reported by parents of young children with autism.

At the time of the research, no single assessment tool eliciting information about the range of comorbid symptoms in young children with autism was available. Likewise, no single measure assessed both physiological and behavioral self-regulatory symptoms. A caregiver assessment of comorbid psychopathology for older children existed (Matson \& Wilkins, 2008), as did several caregiver assessment tools of sensory symptoms in young children (Baranek et al., 2006; Dunn, 1999; JohnsonEcker \& Parham, 2000). Assessment of self-regulation tended to be oriented to the age of the child and the specialty of the examiner; for premature infants, physiological measures were used (temperature, heart rate, etc.); for children with sleep and digestive disorders, parent questionnaires and structured interviews were used (Liu et al., 2006; Valicenti-McDermott et al., 2006); and for older children, attentional, emotional, and behavioral measures were used (Achenbach \& Ruffle, 2000; Aman, Singh, Stewart, \& Field, 1985). To fill this need, we developed the Sense and Self-Regulation Checklist (SSC), a caregiver questionnaire that is divided into items relative to abnormal sensory responses to ordinary injurious and noninjurious stimuli and items relative to difficulties reaching early self-regulation milestones.

We carried out this validation study with three groups of children: children with autism spectrum disorder (ASD), typically developing children, and children with other reasons for developmental delay (other DD). We attempted to answer three main questions:

1. Does the SSC produce reliable scores for sensory and self-regulatory symptoms in the three groups?

2. Does the SSC differentiate between the children with ASD and the other groups on the basis of sensory findings, self-regulatory findings, or both?

3. What is the magnitude of the relationship between comorbid symptoms, as measured by sensory and self-regulatory scores, and severity of autism?

\section{Method}

\section{Participants}

Data from 265 children ages 24-72 mo were collected for this study: 99 children with ASD, 28 children with other $\mathrm{DD}$, and 138 typically developing children. All informants were primary caregivers for the children concerned. The mean age of the ASD group was $3.9 \mathrm{yr}$ (standard deviation $[S D]=1.2) ; 81$ boys and 18 girls were in this group. The mean age in the typically developing group was $3.9 \mathrm{yr}$ $(S D=0.89)$; this group contained 70 boys and 68 girls. The mean age of the other DD group was $2.26 \mathrm{yr}(S D=$ $1.4) ; 17$ boys and 11 girls were in this group. Ages differed significantly across groups, $F(2,263)=28.4, p<.001$; children in the other DD group were younger than those in the other groups.

\section{Data Collection}

We collected SSC data on children with autism from several research projects completed over the past $7 \mathrm{yr}$ with 
institutional review board approval from Western Oregon University. Inclusion criteria for the children with autism were age $<6 \mathrm{yr}$, receiving state-sponsored early childhood special education (ECSE) services for autism, absence of other severe disability such as cerebral palsy, and taking no psychotropic medication. Children were recruited from six regional ECSE programs across Oregon. Diagnosis of autism was confirmed by Louisa M. T. Silva using the criteria in the Diagnostic and Statistical Manual of Mental Disorders (4th ed., text rev.; American Psychiatric Association, 2000).

Data for the other DD group were obtained from a previous study investigating the effect of an intervention strategy on young children with motor developmental delay and motor tone disorders. Inclusion criteria were age $<6 \mathrm{yr}$, presence of clinical hypertonia or hypotonia with motor delay, qualifying for and receiving ECSE physical therapy services, and taking no psychotropic medication. Fifteen children had high tone and were candidates for the diagnosis of cerebral palsy; 10 children had low tone and had Down syndrome. Diagnoses were confirmed by Louisa M. T. Silva through clinical exam and review of medical records.

We recruited parents of typically developing children to complete the surveys from one child care center, three mother support groups, and one toddler drop-in play center in Oregon. Parents completed the surveys on a convenience basis. Inclusionary criteria for the children included age 3-6, no educational or medical diagnosis of autism, absence of DD or previous assessment for DD, and absence of chronic medical conditions.

\section{Measures}

\section{Instrument Development}

The theoretical division of the SSC into Sensory and SelfRegulatory domains comes from a model and treatment for autism that is based on Chinese medicine (Silva, Schalock, \& Ayres, 2011). The SSC was designed for clinical use to identify areas of sensory and self-regulatory difficulty and assess their response to treatment. The individual items were developed and selected through an iterative process by conducting a review of more than 100 interviews of parents with young autistic children in which parents were asked open-ended questions about their child's sensory and self-regulatory responses to ordinary, daily-life situations. As it became evident from parent interviews that sensory responses to both injurious and noninjurious stimuli were grossly abnormal, we created six sensory subdomains: touch-pain, auditory, visual, taste-smell, hyperreactive to noninjurious stimuli, and hyporeactive to injurious stimuli. The hyperreactive and hyporeactive subdomains do not have their own separate items; the hyperreactive subdomain is drawn from all sensory subdomains, and the hyporeactive subdomain is drawn from the touch-pain subdomain only (Table 1).

An additional category for analysis was created in response to parent reports of abnormal touch-pain responses in multiple areas of the body, including the face, head, fingers, toes, skin, and diaper area. The SSC labels touch-pain items by their respective body area, for example, "difficulty with haircuts" is assigned to the head, and "difficulty cutting fingernails" is assigned to the fingers (see Table 1). Information regarding the number of areas involved can be extracted from the Sensory domain and analyzed separately.

Self-regulatory items were informed by Chinese medical theory as well as a knowledge of the normal developmental trajectory for self-regulatory milestones in the first 3 years of life, including self-regulation of sleep, digestion, self-soothing, orientation/attention, and emerging self-regulation of emotions and behavior in response to social cues (Posner \& Rothbart, 2009).

Table 1 lists all SSC items by Sensory and SelfRegulatory domain and identifies which items were extracted to analyze hyporeactivity and hyperreactivity and body part involvement. Items are rated never (0), rarely (1), sometimes (2), or often (3). Domain scores are obtained by summing the individual items. Table 1 also shows the prevalence of responses scored sometimes or often.

Over a 5-yr period, we submitted the SSC to an ongoing development and refinement process in which items were evaluated for inclusion, exclusion, and clarification of terminology. The SSC caregiver report is suitable for use by caregivers who have an elementary school education and is available in English, Spanish, and Chinese.

We used the Pervasive Developmental Disorders Behavior Inventory (PDDBI) Parent Version (Cohen \& Sudhalter, 2005) as an overall measure of the severity of autism. The PDDBI is a two-part rating scale measuring maladaptive behavior and social-communication skills that is designed to assess changes in response to intervention programs in children with pervasive developmental disorder not otherwise specified, autism, or Asperger syndrome. The PDDBI Autism Composite score is based on both parts of the scale. Results are reported in standard scores with a mean of 50 and a standard deviation of 10 .

The PDDBI has gone through extensive development and validation, and external reviews have determined that 


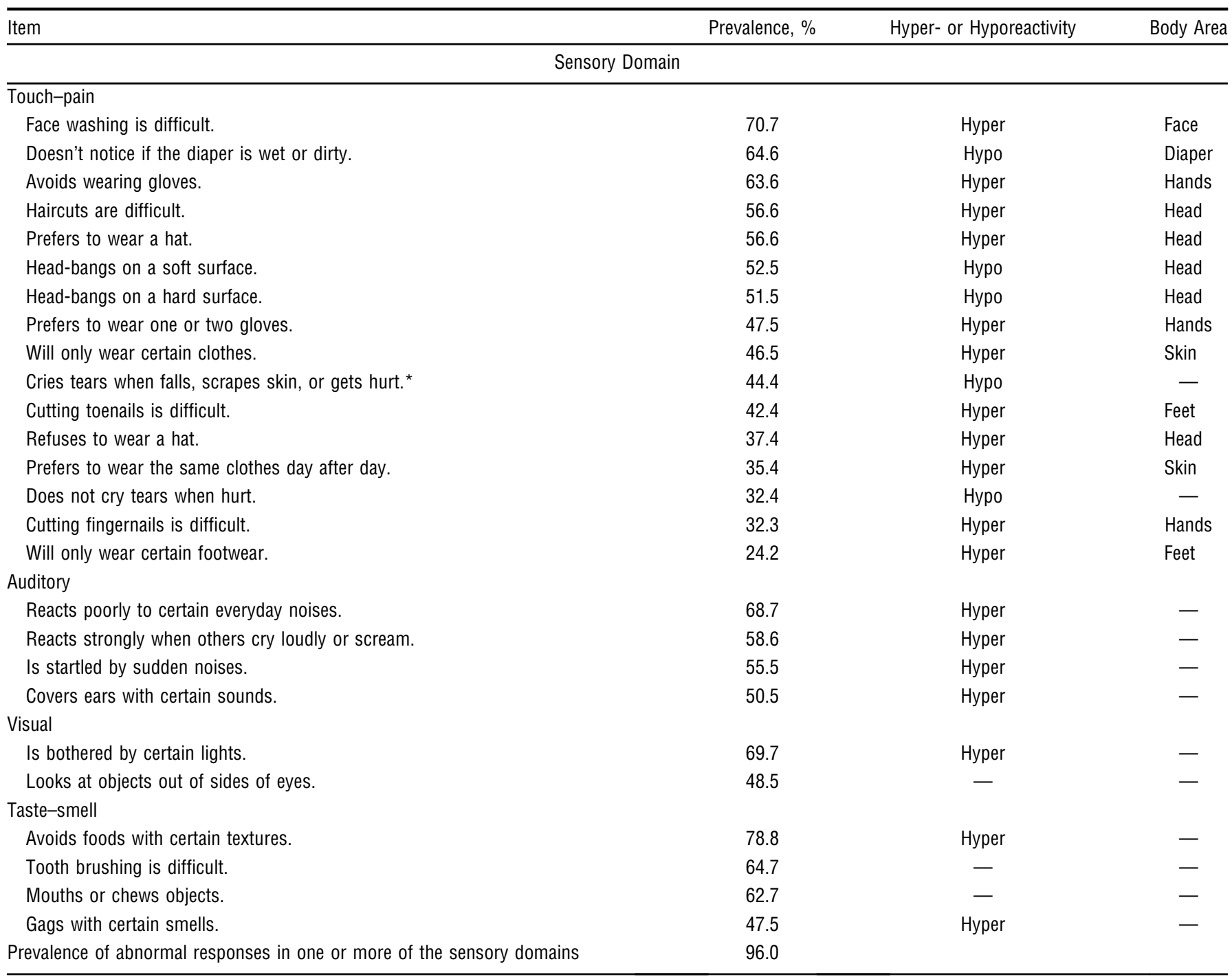

Self-Regulation Domain

\begin{tabular}{|c|c|c|c|}
\hline \multicolumn{4}{|l|}{ Sleep } \\
\hline Has difficulty falling asleep at bedtime. & 57.6 & - & - \\
\hline Awakens very early and stays awake. & 56.6 & - & - \\
\hline Has difficulty falling back asleep when awakens during the night. & 50.5 & - & - \\
\hline Has difficulty awakening in morning. & 34.3 & - & - \\
\hline \multicolumn{4}{|l|}{ Appetite-digestion } \\
\hline Eats very few foods. & 63.6 & - & - \\
\hline Does not seem to be interested in food. & 48.5 & - & - \\
\hline Bowel movement (BM) is every other day. & 38.4 & - & - \\
\hline BM is hard and dry. & 34.3 & - & - \\
\hline Requires regular use of laxative to avoid constipation. & 33.3 & - & - \\
\hline BMs are frequent. & 30.3 & - & - \\
\hline Has difficulty calming him- or herself when upset. & 83.8 & - & - \\
\hline Gets upset or tantrums when asked to make a transition. & 78.7 & - & - \\
\hline Tantrums or meltdowns. & 45.5 & - & - \\
\hline Cries easily when frustrated. & 32.3 & - & - \\
\hline
\end{tabular}




\begin{tabular}{lcc}
\hline Item & Prevalence, \% & Hyper- or Hyporeactivity \\
\hline Orienting-attending & & Body Area \\
Has to be prompted to make eye contact when spoken to. & 74.7 & - \\
Stares off into space. & 70.7 & - \\
Seems not to notice when spoken to in a normal voice. & 67.6 & - \\
Does not notice or react when tapped on the back. & 65.7 & - \\
Does not respond to his or her name. & 64.6 & - \\
Aggressive behavior & 58.5 & - \\
Hits or kicks others. & 56.5 & - \\
Bites others. & 52.5 & - \\
Scratches or pulls others' hair. & 46.6 & - \\
Throws things at others. & & - \\
Self-injurious behavior & 49.5 & - \\
Hits self. & 39.4 & - \\
Bites self. & 31.3 & - \\
Pulls own hair. & 98.0 & - \\
Prevalence of significant difficulties in one or more of the self-regulatory domains & - \\
\hline
\end{tabular}

Note. - = not applicable.

${ }^{\star}$ Scores for rating this item are reversed.

it demonstrates construct and criterion validity sufficient for use in research. The PDDBI has also been found to be reliable and has high levels of internal consistency ( $\alpha$ s $=$ $.80-.98$ across the various domains and constructs).

\section{Results}

\section{Question 1: Reliability of the SSC}

Both internal consistency and test-retest stability were assessed for the SSC. We calculated internal consistency estimates (Cronbach's $\alpha$ ) for the total SSC score, the Sensory and Self-Regulation domain, and the individual subdomains for each population. Domain and overall scale scores demonstrate acceptable internal consistency across all three groups. These results are shown in Table 2. Overall $\alpha$ s were .87 for children with ASD, .89 for typically developing children, and .85 for children with other DD. In the Sensory domain, $\alpha$ s were .81 for ASD, .80 for typically developing, and .58 for other DD. For the Self-Regulation domain, $\alpha s$ were .79 for ASD, .86 for typically developing, and .83 for other DD. Subdomain scores demonstrated acceptable internal consistency across all three groups in the subdomains of self-soothing and aggressive behavior. Test-retest stability estimates were calculated for the total SSC score and the Sensory and Self-Regulation domains with a subsample of parents of 38 children with autism at a 4-month interval. The test-retest coefficient for sensory impairment was .595; mean scores (44.74 and 43.37) were stable over assessments. The test-retest coefficient for self-regulation was .831; mean scores (36.29 and 36.45) also were stable over assessments. For the overall score, the test-retest coefficient was .677 , with mean scores (84.0 and 82.50) stable over assessments.

\section{Question 2: Differentiation Between the Children With ASD and Other Groups}

The SSC's ability to discriminate on the basis of frequency or intensity of abnormal responses as evaluated with analyses of variance (ANOVAs) is shown in Table 3. The group differences for the total sensory scores $(F[2,262]=124, p<$ $.001)$, total self-regulatory scores $(F[2,262]=122, p<$ $.001)$, and total sensory and self-regulatory scores $(F[2$, $262]=151, p<.001)$ were highly significant. To determine whether gender or age confounded the findings for each disability group, we ran separate two-way ANOVAs. Neither gender nor age was found to be significantly related to either sensory impairment or self-regulation. For sensory impairment, neither gender $(F[1,263]=0.05, p=.826)$ nor the Gender $\times$ Disability interaction $(F[2,262]=0.50$, $p=.606]$ was significant. For age, neither age $(F[4,260]=$ $0.34, p=.914)$ nor the Age $\times$ Disability interaction $(F[7$, $257]=0.54, p=.800)$ was significant. For self-regulation, regarding gender, neither gender $(F[1,263]=0.60, p=$ $.551)$ nor the Gender $\times$ Disability interaction $(F[2,262]=$ $0.03, p=.865)$ was significant. For age, neither age $(F[4$, $260]=0.71, p=.645)$ nor the Age $\times$ Disability interaction $(F[7,257]=0.61, p=.747)$ was significant.

Post hoc Scheffé test comparisons of the ASD and typically developing subdomains showed that the SSC differentiated in six of six sensory and six of six self-regulatory subdomains. Post hoc Scheffé test comparisons between the ASD and other DD subdomains showed that the SSC differentiated three of six sensory subdomains (touchpain, hyporeactivity, and hyperreactivity) and five of six self-regulatory domains (sleep, digestion, self-soothing, orientation-attention, and self-injurious behavior). We 


\begin{tabular}{|c|c|c|c|c|}
\hline \multirow[b]{2}{*}{ Domain } & \multicolumn{3}{|c|}{ Cronbach's $\alpha$ Reliability } & \multirow[b]{2}{*}{ No. of Items } \\
\hline & ASD & Typically Developing & Other DD & \\
\hline Sensory domain & .812 & .795 & .579 & 26 \\
\hline Tactile & .749 & .687 & .468 & 16 \\
\hline Auditory & .720 & .748 & .586 & 4 \\
\hline Visual & .527 & .455 & .844 & 2 \\
\hline Taste and smell & .355 & .396 & .346 & 4 \\
\hline Hyporeactivity & .528 & .259 & .0 & 4 \\
\hline Hyperreactivity & .795 & .786 & .596 & 19 \\
\hline Self-Regulation domain & .785 & .855 & .828 & 30 \\
\hline Sleep & .577 & .551 & .707 & 4 \\
\hline Digestion & .554 & .749 & .360 & 10 \\
\hline Self-soothing & .820 & .752 & .877 & 4 \\
\hline Orientation and attention & .818 & .720 & .543 & 5 \\
\hline Behavior-aggression & .786 & .803 & .827 & 4 \\
\hline Behavior-self-injurious & .702 & .002 & .816 & 3 \\
\hline Total sensory + self-regulation & .873 & .894 & .850 & 56 \\
\hline
\end{tabular}

Note. ASD = autism spectrum disorder; DD = developmental delay.

found no significant differences between the ASD and other DD groups in the subdomains of visual, auditory, taste-smell, or aggressive behavior.

We also investigated the SSC's ability to discriminate between the ASD and other DD groups on the basis of the number of senses involved and the number of areas of body involvement by analyzing frequency distributions using $\chi^{2}$ analyses and the median test. These distributions are shown in Table 4.

To determine the prevalence of abnormal sensory responses for each sense, we set the criterion for abnormal sensory response as the mean score for typically developing children plus 1 standard deviation (Baranek et al., 2006). As expected, children with ASD had a higher prevalence of abnormal responses in the individual senses than did children in the other groups. For example, $76 \%$ of children with ASD had tactile impairments compared with $50 \%$ of children with other DD and $17 \%$ of typically developing children. The $\chi^{2}$ analyses on the four sensory domains indicate significant differences in distributions (tactile $\chi^{2}[2, N=265]=83.2, p<.001$; visual $\chi^{2}[2$, $N=265]=48.6, p<.001$; auditory $\chi^{2}[2, N=265]=$ 54.8, $p<.001$; taste-smell $\chi^{2}[2, N=265]=90.7, p<$ $.001)$. To determine whether the number of senses affected differed across the groups, we conducted a median test, which indicated that groups differed on the number of senses affected $\left(\chi^{2}[2, N=265]=99.2, p<.001\right)$. The children with ASD were more multisensorily impaired than the children in other groups.

We conducted a parallel analysis on areas of the body affected. Although the results were not consistent across all areas of the body, children with ASD had a higher prevalence of abnormal responses in more areas of the body than did the other groups. The $\chi^{2}$ analyses on the six body areas indicate the following significant differences in distributions: face, $\chi^{2}(2, N=265)=66.3, p<.001$; head, $\chi^{2}$ $(2, N=265)=34.8, p<.001$; hands, $\chi^{2}(2, N=265)=$ 14.7, $p<.01$; feet, $\chi^{2}(2, N=265)=12.3, p<.01$; skin, $\chi^{2}$ $(2, N=265)=18.9, p<.001$; diaper, $\chi^{2}(2, N=265)=$ 73.7, $p<.001$. To determine whether the number of body areas affected differed across the groups, we conducted a median test, which indicated that groups differed on the number of areas of the body affected $\left(\chi^{2}[2, N=265]=\right.$ 65.7, $p<.001)$. The children with ASD were affected in more areas of the body than other groups.

\section{Question 3: Magnitude of the Relationship Between Comorbid Sensory and Self-Regulatory Symptoms}

The PDDBI Autism Composite scores were correlated with both sensory impairment and self-regulatory difficulty scores. These relationships are shown in Supplemental Figures 1 and 2 (available online at http://ajot. aotapress.net; navigate to this article, and click on "Supplemental Materials"). Positive and relatively strong relationships between sensory impairment and severity of autism $(r=.543)$ and self-regulation impairment and severity of autism $(r=.418)$ exist. Higher levels of sensory and self-regulation impairment are related to more severe levels of autism. Both correlations are significant at the .001 level. Scores on the PDDBI cluster at the upper end of the range; a score of 50 represents a "typical" child with autism.

We conducted multiple regression to further study the relationship between sensory and self-regulation 


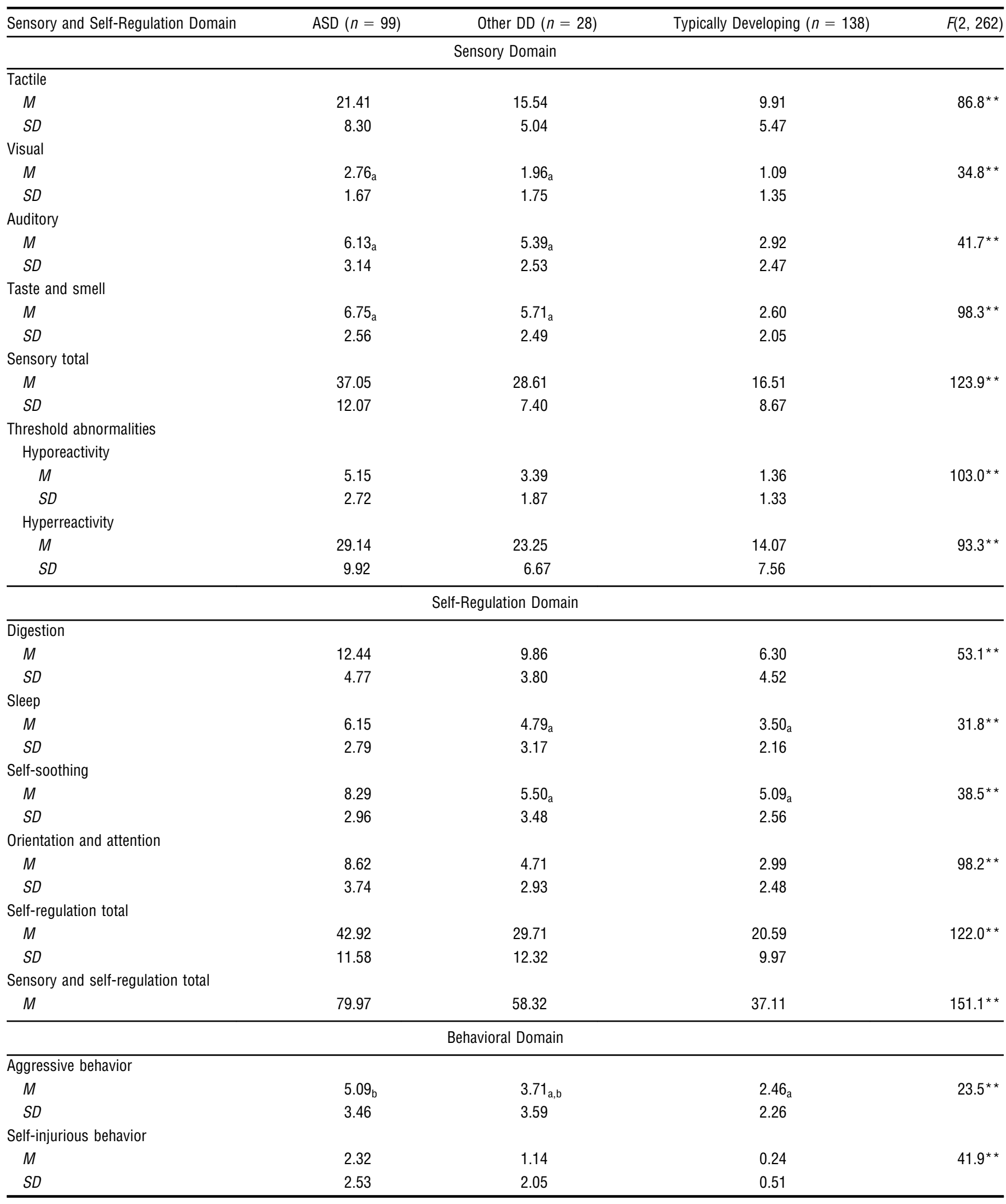

Note. Means sharing common subscript letters a and $\mathrm{b}$ are not significantly different. ASD = autism spectrum disorder; DD = developmental delay; $M=$ mean; $S D=$ standard deviation.

${ }^{\star *} p<.001$, two-tailed.

impairment and severity of autism. The regression coefficient for sensory impairment was $.402(p<.01)$. The regression coefficient for self-regulation was .188 (not significant). The overall model was significant $(F[2,58]=$ $13.79, p<.001$ ), with an $R^{2}$ value of .322 . One-third of the variance in PDDBI Autism Composite scores is 


\begin{tabular}{lcccc}
\hline \multirow{2}{*}{ Group } & \multicolumn{4}{c}{ Sensory Domain Impaired, \% } \\
\cline { 2 - 5 } & Tactile & Visual & Auditory & Taste and Smell \\
\hline ASD & 75.8 & 57.6 & 58.6 & 79.8 \\
Other DD & 50.0 & 35.7 & 50.0 & 71.4 \\
Typically developing & 16.7 & 14.5 & 13.8 & 20.3 \\
\hline
\end{tabular}

\begin{tabular}{lrrrrc}
\hline & \multicolumn{5}{c}{ No. of Senses Impaired } \\
\cline { 2 - 6 } & \multicolumn{1}{c}{4} & 3 & 2 & 1 & 0 \\
\hline ASD & 32.3 & 29.3 & 20.2 & 14.1 & 4.0 \\
Other DD & 7.1 & 28.6 & 32.1 & 28.6 & 3.6 \\
Typically developing & 0.7 & 7.2 & 10.1 & 20.3 & 61.6
\end{tabular}

\begin{tabular}{lcccccc}
\hline & \multicolumn{5}{c}{ Areas of the Body Affected } \\
\cline { 2 - 6 } & Face & Head & Hands & Feet & Skin & Diaper \\
\hline ASD & 69.7 & 65.7 & 34.3 & 42.4 & 64.6 \\
Other DD & 57.1 & 50.0 & 57.1 & 46.4 & 17.9 & 27.1 \\
Typically developing & 18.1 & 19.6 & 21.7 & 23.2 & 21.7 \\
\hline
\end{tabular}

No. of Areas of the Body Affected

\begin{tabular}{|c|c|c|c|c|c|c|}
\hline & \\
\hline & 6 & 5 & 4 & 3 & 2 & 1 \\
\hline Other DD & 0 & 21.4 & 25.0 & 7.1 & 21.4 & 14.3 \\
\hline
\end{tabular}

Note. $\mathrm{ASD}=$ autism spectrum disorder; $\mathrm{DD}=$ developmental delay.

explained by the variance in sensory and self-regulation scores.

\section{Discussion}

This study demonstrates that the SSC has adequate psychometric validity to measure sensory and self-regulation problems in young children with ASD and to discriminate such children from other groups. As such, the SSC is unique and useful to clinicians and researchers. SSC sensory findings converge with previously published research showing that abnormal sensory responses in autism are different from those in other groups-they are more severe and multisensory and characterized by more hyporeactivity and hyperreactivity. These findings have been reported using the Sensory Experiences Questionnaire (Baranek et al., 2006) and the Sensory Profile (Ben-Sasson et al., 2009). These findings have been quantified in adults with autism with quantitative sensory testing, and abnormal sensory thresholds to gentle touch (Cascio et al., 2008), pain, temperature, and low-frequency vibration (Blakemore et al., 2006), as well as hypoesthesia (reduced sensitivity to injurious stimuli) and hyperesthesia (painful response to noninjurious stimuli), have also been reported.

SSC self-regulatory findings in individual domains converge with findings reported for sleep using the Children's Sleep Habits Questionnaire (Malow et al., 2006), for digestion using structured interviews (Valicenti-
McDermott et al., 2006), and for attention and behavior using the Child Behavior Checklist (Hartley et al., 2008).

Sensory and self-regulatory findings diverge from published research in reporting two new findings discriminating children with ASD from children with other reasons for developmental delay as well as from typically developing children: (1) tactile sensory impairment, present on multiple areas of the body and characterized by hypersensitivity to noninjurious stimuli and hyposensitivity to injurious stimuli, and (2) the presence of global selfregulatory delay. This study is the first time that a discrete abnormality of touch in a setting of multisensory impairment has been shown to discriminate autism from other groups. And because the SSC makes it possible to evaluate all the early self-regulation milestones in one instrument, our study is also the first time that children with autism have been discriminated from other groups by the presence of global delay of early self-regulatory milestones. The SSC reports a prevalence for sensory and self-regulatory symptoms that approaches $100 \%$ (96\% for sensory and $98 \%$ for self-regulatory; see Table 1). This prevalence is higher than has previously been reported with other instruments and begs the consideration that sensory and self-regulatory symptoms represent an integral part of autism rather than a comorbid group of symptoms, as currently considered.

The degree of significance distinguishing children with ASD from other groups on the basis of tactile findings 
related to each of the six areas surveyed (i.e., face, head, hands, feet, skin, diaper area) is quite remarkable $(p<.001$ for five of the six areas), as is the degree of significance distinguishing children with ASD by number of areas involved $(p<.001)$. The tactile findings are compatible with peripheral neuropathy, a condition of damage to the peripheral sensory and autonomic nerve fibers characterized by hypoesthesia to injurious stimuli and hyperesthesia to noninjurious stimuli, often present in multiple locations of the body (Baron, 2009). Earlier studies have demonstrated abnormal quantitative sensory testing in people with autism (Blakemore et al., 2006; Cascio et al., 2008). Given the critical importance of touch to early social development demonstrated in orphan studies (Chrisholm, 1998) and the yet-unknown mechanism for the delay of social development seen in autism, these qualitative findings offer a potential explanation for the social delay in autism and should be further investigated in a larger sample with quantitative sensory testing.

The finding that the autism group was differentiated by a global delay of early self-regulatory milestones also offers an explanation for how social delay might develop in autism. Early self-regulatory abilities are a cornerstone of early childhood development that cut across all domains (Shonkoff \& Phillips, 2000). As such, early social abilities are built upon an earlier foundation of self-regulatory abilities. It is almost self-evident that delay of early selfregulatory abilities is likely to be associated with subsequent delay of social abilities, as was the case here. For practitioners familiar with the clinical presentation of young children with autism, this concept can be illustrated by trying to imagine a 3-year-old child with normal self-regulatory abilities (i.e., eating and sleeping normally, self-soothing appropriately, orienting and paying attention, having the emerging ability to regulate behavior and emotions in response to social cues) who also has autism. As the exercise illustrates, this is difficult to imagine because the social milestones that are delayed in autism arise out of the self-regulatory milestones relative to orientation/attention, self-soothing, and the emerging ability to regulate behavior and emotions in response to social cues.

How and why abnormal sensory responses and selfregulatory delays are related to each other remains to be explored for the three groups of children. This exploration is beyond the scope of this validation study but is the focus in a separate article (Silva \& Schalock, 2011).

\section{Implications for Occupational Therapy Practice}

Our study has the following implications for occupational therapy practice:
- The SSC is a valid measure of sensory and self-regulatory difficulties for children under age 6 .

- The SSC can be used as a treatment outcomes measure for children with autism.

- The SSC can also be used to identify patterns of sensory and self-regulatory difficulties in young children with other disabilities.

\section{Limitations and Future Research}

This study has several limitations. First, larger samples are needed to achieve acceptable internal consistency in the subdomain scores across groups to satisfactorily control for variability in symptoms. In particular, a larger other DD group is of interest to further differentiate children with ASD from children with other intellectual or developmental disabilities. Second, mental and developmental age data need to be collected and correlated with SSC scores to place the findings in a more clinical context. Third, collecting more demographic data on the informants answering the SSC would be of interest. A larger research study addressing these limitations is planned.

\section{Acknowledgments}

We gratefully acknowledge the families and early intervention programs that participated in our research. In addition, we thank the Curry Stone Foundation and Northwest Health Foundation, whose generous support made this research possible. Moreover, we acknowledge the data management work on the project by Nancy Ganson and Sharon Kadell, as well as the project management and editing work of Kristen Gabrielsen.

\section{References}

Achenbach, T. M., \& Ruffle, T. (2000). The Child Behavior Checklist and related forms for assessing behavioral/emotional problems and competencies. Pediatrics in Review, 21, 265-271.

Aman, M. G., Singh, N. N., Stewart, A. W., \& Field, C. J. (1985). The Aberrant Behavior Checklist: A behavior rating scale for the assessment of treatment effects. American Journal of Mental Deficiency, 89, 485-491.

American Psychiatric Association. (2000). Diagnostic and statistical manual of mental disorders (4th ed., text rev.). Washington, DC: Author.

Baranek, G. T., David, F. J., Poe, M. D., Stone, W. L., \& Watson, L. R. (2006). Sensory Experiences Questionnaire: Discriminating sensory features in young children with autism, developmental delays, and typical development. Journal of Child Psychology and Psychiatry and Allied Disciplines, 47, 591-601. http://dx.doi.org/10.1111/j.1469-7610.2005.01546.x

Baron, R. (2009). Neuropathic pain: A clinical perspective. In B. J. Canning \& D. Spina (Eds.) \& F. B. Hofmann et al. (Series Eds.), Handbook of experimental pharmacology: Vol. 194. Sensory nerves (pp. 3-30). New York: Springer. 
Ben-Sasson, A., Hen, L., Fluss, R., Cermak, S. A., Engel-Yeger, B., \& Gal, E. (2009). A meta-analysis of sensory modulation symptoms in individuals with autism spectrum disorders. Journal of Autism and Developmental Disorders, 39, 1-11. http://dx.doi.org/10.1007/s10803-008-0593-3

Blakemore, S. J., Tavassoli, T., Calò, S., Thomas, R. M., Catmur, C., Frith, U., et al. (2006). Tactile sensitivity in Asperger syndrome. Brain and Cognition, 61, 5-13. http://dx.doi.org/10.1016/j.bandc.2005.12.013

Cascio, C., McGlone, F., Folger, S., Tannan, V., Baranek, G., Pelphrey, K. A., et al. (2008). Tactile perception in adults with autism: A multidimensional psychophysical study. Journal of Autism and Developmental Disorders, 38, 127-137. http://dx.doi.org/10.1007/s10803-007-0370-8

Chrisholm, K. (1998). A three year follow-up of attachment and indiscriminate friendliness in children adopted from Romanian orphanages. Child Development, 69, 1092-1106.

Cohen, I. L., \& Sudhalter, V. (2005). PDD Behavior Inventory. Lutz, FL: Psychological Assessment Resources.

Dunn, W. (1999). The Sensory Profile: Examiner's manual. San Antonio, TX: Psychological Corporation.

Hartley, S. L., Sikora, D. M., \& McCoy, R. (2008). Prevalence and risk factors of maladaptive behaviour in young children with autistic disorder. Journal of Intellectual Disability Research, 52, 819-829. http://dx.doi.org/10.1111/j.13652788.2008.01065.x

Ibrahim, S. H., Voigt, R. G., Katusic, S. K., Weaver, A. L., \& Barbaresi, W. J. (2009). Incidence of gastrointestinal symptoms in children with autism: A population-based study. $\mathrm{Pe}-$ diatrics, 124, 680-686. http://dx.doi.org/10.1542/peds.20082933

Johnson-Ecker, C. L., \& Parham, L. D. (2000). The evaluation of sensory processing: A validity study using contrasting groups. American Journal of Occupational Therapy, 54, 494-503. http://dx.doi.org/10.5014/ajot.54.5.494

Kern, J. K., Trivedi, M. H., Garver, C. R., Grannemann, B. D., Andrews, A. A., Savla, J. S., et al. (2006). The pattern of sensory processing abnormalities in autism. Autism, 10, 480-494. http://dx.doi.org/10.1177/1362361306066564

Leekam, S. R., Nieto, C., Libby, S. J., Wing, L., \& Gould, J. (2007). Describing the sensory abnormalities of children and adults with autism. Journal of Autism and Developmental Disorders, 37, 894-910. http://dx.doi.org/ 10.1007/s10803-006-0218-7

Levy, S. E., Mandell, D. S., \& Schultz, R. T. (2009). Autism. Lancet, 374, 1627-1638. http://dx.doi.org/10.1016/S01406736(09)61376-3

Liu, X., Hubbard, J. A., Fabes, R. A., \& Adam, J. B. (2006). Sleep disturbances and correlates of children with autism spectrum disorders. Child Psychiatry and Human Development, 37, 179-191. http://dx.doi.org/10.1007/s10578-0060028-3

Malow, B. A., Marzec, M. L., McGrew, S. G., Wang, L., Henderson, L. M., \& Stone, W. L. (2006). Characterizing sleep in children with autism spectrum disorders: A multidimensional approach. Sleep, 29, 1563-1571.
Matson, J. L., \& Wilkins, J. (2008). Reliability of the Autism Spectrum Disorders-Comorbid for Children (ASD-CC). Journal of Developmental and Physical Disabilities, 20, 327-336. http://dx.doi.org/10.1007/s10882-008-9100-1

Nikolov, R. N., Bearss, K. E., Lettinga, J., Erickson, C., Rodowski, M., Aman, M. G., et al. (2009). Gastrointestinal symptoms in a sample of children with pervasive developmental disorders. Journal of Autism and Developmental Disorders, 39, 405-413. http://dx.doi.org/10.1007/s10803008-0637-8

Posner, M. I., \& Rothbart, M. K. (2009). Toward a physical basis of attention and self-regulation. Physics of Life Reviews, 6, 103-120. http://dx.doi.org/10.1016/j.plrev.2009.02.001

Reebye, P. N., \& Elbe, D. (2009). The role of pharmacotherapy in the management of self-regulation difficulties in young children. Journal of the Canadian Academy of Child and Adolescent Psychiatry, 18, 150-159.

Shonkoff, J. P., \& Phillips, D. A. (Eds.). (2000). From neurons to neighborhoods: The science of early childhood development. Washington DC: National Academy Press.

Silva, L. M. T., \& Schalock, M. (2011). Positive relationship between early self-regulatory difficulties and abnormal sensory threshold responses in preschool children. Manuscript submitted for publication.

Silva, L. M. T., Schalock, M., \& Ayres, R. (2011). A model and treatment for autism at the convergence of Chinese medicine and Western science: First 130 cases. Chinese Journal of Integrative Medicine, 17, 421-429.

Silva, L. M. T., Schalock, M., Ayres, R., Bunse, C., \& Budden, S. (2009). Qigong massage treatment for sensory and self-regulation problems in young children with autism: A randomized controlled trial. American Journal of Occupational Therapy, 63, 423-432. http://dx.doi. org/10.5014/ajot.63.4.423

Silva, L. M. T., Schalock, M., \& Gabrielsen, K. (2011). Early intervention for autism with a parent-delivered qigong massage program: A randomized controlled trial. American Journal of Occupational Therapy, 65, 550-559. http://dx. doi.org/10.5014/ajot.2011.000661

Souders, M. C., Mason, T. B. A., Valladares, O., Bucan, M., Levy, S. E., Mandell, D. S., et al. (2009). Sleep behaviors and sleep quality in children with autism spectrum disorders. Sleep, 32, 1566-1578.

Tordjman, S., Anderson, G. M., Botbol, M., Brailly-Tabard, S., Perez-Diaz, F., Graignic, R., et al. (2009). Pain reactivity and plasma $\beta$-endorphin in children and adolescents with autistic disorder. PLoS ONE, 4, e5289. http://dx.doi.org/10.1371/ journal.pone.0005289

Valicenti-McDermott, M., McVicar, K., Rapin, I., Wershil, B. K., Cohen, H., \& Shinnar, S. (2006). Frequency of gastrointestinal symptoms in children with autistic spectrum disorders and association with family history of autoimmune disease. Journal of Developmental and Behavioral Pediatrics, 27(2, Suppl.), S128-S136. http://dx.doi.org/10.1097/00004703200604002-00011

Yanchi, L. (1988). The essential book of traditional Chinese medicine: Vol. 1.Theory. New York: Columbia University Press. 